\title{
电力调度自动化技术研究
}

代兵

国网河南省西华县供电公司

DOI:10.32629/hwr.v2i12.1771

[摘 要] 电力调度自动化系统是指直接为电网运行服务的数据采集与监控系统,包括在此系统运行的应用软件, 是在线为各 级电力调度机构生产运行人员提供电力系统运行信息、分析决策工具和控制手段的数据 处理系统。

[关键词] 电力调度; 自动化系统; 罙余容错结构; 网络几余热备份

电力系统调度控制的基本任务在于保证系统的安全运 行, 以质量合格的电能满足用户用电的需要, 并使发电成本 为最低。传统的电力系统调度控制中心需要采集和处理的数 据数量多, 实时性差, 已无法满足要求。特别是在事故情况下, 丧失时机可能就会造成极大的危害。科学技术的发展为调度 自动化提供了有力的支持, 使用电子计算机对电力系统进行 监视和控制实现电网调度自动化系统, 调度自动化系统收 集、处理电网运行实时信息, 通过人机联系系统把电网运行 状况集中而有选择的显示出来进行监控。运行人员可借此统 观全局, 集中全力指挥全网安全、经济和优质运行。

\section{1 电力调度自动化系统设计}

电力调度自动化系统目标在于提高电网安全运行水平, 提高故障恢复能力、切实减少损失。调度自动化系统应使其 具有数据采集和监视、控制 (SCADA) 的功能, 但在具体实施过 程中应根据调度职责范围、调度自动化现状、基础设备自动 化条件, 按照由低至高的、由易到难的原则恰当确定总体功 能。

系统实现开放的全组态的调度综合自动化系统, 不但使 画面, 后台语言具有可组态性, 而且使数据采集设计也具有 可组态性, 实现以上能力的根本原因在于系统抽象了设备通 道和通讯规约层次。

系统实现基于用户的要求构造满意的人机界面, 常用的 构造人机界面的美工工具都具有动画属性, 包括 “颜色、大 小、位置、文本、角度、闪炼、隐藏” 等属性, 这些属性可 以简单地与以监控变量或内存变量为参数的表达式连接, 系 统提供高效的画面刷新机制, 保证图形元素属性随变量表达 式属性的变化而变化; 系统提供独立功能的控件用于显示系 统的报警信息, 曲线棒图等, 这些控件也可以以某种单位与 监控点变量或表达式及其历史数据库连接, 完成有关的查询 和显示功能。系统的基本图形元素、控件等还可形成满意的 人机界面, 达到对 RTU、智能仪表、板卡以及程序流程等的 控制, 可以达到采用通用语言编程的效果和满意程度。

\section{SCADA 系统应用}

为了提高系统的可靠性, 自动化系统主站网络采用以太 网结构、主机工作站、前置机和网络服务器均通过各自所配
网卡的 RJ-45 插座连至网络集线器 (HUB) 上, 双机切换柜分 别与两台前置机中的多口智能接口相连, MODEM 与双机切换 柜线中一对一相连, 具体提升措施如下:

2.1 网络形式多种多样, 如 Ether Net、FDDI 或 ATM 等 都可使用。

2.2 单网、双网、低速网、高速网可以任意方式进行组 合。系统支持灵活的网络配置, 可以是单低速网、单高速网, 可以是低速和高速双网混用, 也可以是双高速网。

2.3 采取网络咒余热备份。系统正常运行时, 两个网络 上都传输有用数据, 并且两个网络上的数据流量保持动态平 衡。当一个网络工作不正常时, 系统将自动地通过另一网络 传输所有数据。当故障网络恢复正常时, 双网络将自动恢复 到流量的动态平衡状态。从严格意义上来说, 此系统的网络 切换实际上是网络传输功能的弹性伸缩, 网络本身对系统是 透明的, 双网络并无主、备之分。

2.4 支持标准的网络接口, 可以方便地与其它系统如 MIS 等进行互联。

2.5 易于与上级或下级调度组成广域网, 进行网络数据 交换, 支持远程调试。在数据库连接技术方面, SCADA 系统也 采取相关措施, 主要体现在如下四个方面: (1) 支持组态地将 系统实时数据库按用户指定的周期或事件产生触发刷新用 户指定的外部实时数据库; (2) 支持直接读写指定数据库记 录的字段数据, 并具备将该数据与该系统组态定义的变量对 应连接的能力, 这使得该系统可以通过数据库与其它任何支 持数据访问的应用程序实时交换信息; (3) 通过标准 SQL 语 句完成外部数据库的一般维护操作, 如建表、删除表、插入、 修改和删除记录; (4) 通过后台 API 的方式, 将电力自动化系 统中的常用的数据库查询工作打包, 用户无需编写有关 SQL 语句, 只要简单地提供符合常规应用习惯的参数即可完成复 杂的历史数据库查询和浏览工作。

\section{3 系统性能指标优化}

3.1 系统采取冗余容错结构

系统实现双网络容错是真正的热备用, 双网络正常运行 时, 主、备网络同时都传送有用系统数据, 双网络上的数据流 量保持动态的平衡。 
系统采取双服务器方式, 当系统配置了主备服务器后, 每个客户端同时与两个服务器连接, 并向两个服务器发送信 息, 服务器控制程序自动检测客户端与服务器的连接模式, 以确保唯一的数据转发, 或将有关信息转发到感兴趣的客户 端。同时客户端也自动检测服务器的状态。

系统采取双前置机方式:（1）基于 485 总线方式的双机 切换; (2) 基于 Nport Server 的双机切换; (3) 用户自定义 方式的双机切换。系统采取双通道方式: (1)系统采取以通道 的方式与 RTU 等采集设备进行连接; (2)系统支持自动主备通 道切换, 不支持手动切换, 并且是采用冷备用原理。当主通道 在传输数据时, 备用通道不采集数据。当系统检测到主通道 连接出现故障或者误码率过高, 则自动启动备用通道采集数 据, 并将停止主通道的采集, 此时主通道的地位转变为备用 通道, 原备用通道变为主通道不能重新接管数据的采集工作, 除非当前的主通道出现故障。

\section{2 系统采取的网络通讯结构}

采用点对点通讯模型主动传输系统改变的实时数据。网 络环境下, 实时数据库数据项的改变有以下三种可能: 从通 道采集数据改变实时数据库; 运行后台语言实时数据库; 从 网络其它节点传递来改变实时数据库。采用客户/服务器查 询方式, 在网络中传递历史数据和进行实时数据库状态恢 复。

系统对历史数据采用客户/服务器方式, 在实际应用中, 如对 SOE 的查询、对历史曲线的查询等操作中, 一般是用户 提交查询条件, 由系统将有关查询条件变为连接的历史数据 库能够接受的标准或非标准 SQL 语句, 提交给数据库服务器, 从历史数据库中查询得到满足有关条件的查询结果集, 数据 库服务器将该结果集通过网络传递给查询的计算机, 计算机 运行系统根据接收到的查询结果, 将它转变为用户容易理解 的方式, 如曲线、报表等显示出来。

系统利用网络协议实现方便的容错系统模型, 在该模型 中, 运行系统采用总线方式或通过专门的切换装置与连接的 RTU 或其它智能数据采集设备连接, 当主系统出现故障或通 道出现故障时, 备用系统将自动或手动获得控制权, 保证系 统正常运行。

3.3 实现网络构架的有效扩充

(1) 架设远程工作站。正常情况下所有计算机都是通过
各自所配置的 $10 \sim 100 \mathrm{M}$ 网卡连至集线器上,传输媒质选择 的是 8 芯双绞线, 这样的组网如果在两座比较分散的建筑物 之间 (线距 $1.5 \mathrm{~km}$ 以上), 则信号的抗干扰能力、准确度、保 密能力都会大为下降, 对准确度、实时性要求较高的工作站 来讲, 也就是说必须架设能满足的远程工作站, 以解决距离 服务器较远部门和系统的连网问题。(2) 架设移动工作站。 移动工作站的性质和远程工作有相似之处, 而且有可移动性, 其架设更有必要性。系统的原始数据、通道及远端接口都进 行定期测试, 传统的测试方法是部分人员在现场测量数据、 计算结果, 后台人员电话核对显示值和测试值, 这样在准确 性、及时性方面会受到很大影响, 如果携带移动工作站至现 场, 在测试时由移动站向后台服务器请求数据与所测数据核 对, 准确度可得到较好的保障, 其灵活性、实时性也非人眼可 比。从移动站直接观测后台数据的同时, 可以通过 RTU 的 RS-232 接口观察输出数据, 并能直接进行遥控、遥测实验。 管理人员外出时, 如果携带移动工作站, 只要拨号和中心站 连接, 就可以方便的查看电网信息, 了解系统情况。(3) 实现 远程维护。在传统情况下, 当客户的软硬件系统出现故障时, 通常需要厂家技术人员到现场维护, 这种维护方式实时性 差、效率低, 还会造成用户停机过长, 可能造成很大损失。计 算机远程维护系统通过传输媒质和中心站连接, 技术人员从 自己的维护工作站对自动化系统的故障点进行分析判断, 实 现异地在线调试、修改和升级; 同时还能进行目录查看、文 件图像传输、实时语言对话。

\section{4 结语}

本文对电力调度自动化系统性能、可靠性的提升, 提出 了相关针对性措施, 有助于切实提高电网安全运行水平, 提 高处理事故能力, 减少停电损失, 具有较强的实际应用参考 价值。

\section{[参考文献]}

[1]王伟佳.电网调度自动化系统的发展方向探析[J].科 技信息,2009(19):317.

[2]吴昊琛. 探究电力调度自动化系统应用现状与发展趋 势[J].中小企业管理与科技(上旬刊),2009(6):246.

[3]曹铁生.电力调度自动化系统应用现状与发展趋势研 究分析[J].硅谷,2014(23):74. 